\title{
Probabilistic Approach for Blocking Probability in Optical Networks
}

\author{
Robin Khurana \\ Student \\ SSIET \\ Derabassi, Punjab, India
}

\author{
Ashish Verma \\ Assistant Professor \\ SSIET \\ Derabassi, Punjab, India
}

\author{
Gaganpreet Kaur \\ Assistant Professor \\ SSIET \\ Derabassi, Punjab, India
}

\begin{abstract}
An optical network is a communication network in which information is transmitted as optical signals. In network data traverse through different routes, so deciding best route becomes an important issue in optical network. To select best route in the network various algorithms proposed by researchers. Blocking probability, routing etc are the various parameters related to these algorithms. For calculating the results for these parameters Erlang B formula was used. This paper presents a proposal of mathematical model to reduce the blocking probability in the network. The mathematical model is modification of Erlang's B formula. MATLAB software is used for simulation. Results show that, with increase in the value of load, blocking probability increases but negligibly small. The results have proved that this model is better than the conventional techniques.
\end{abstract}

\section{General Terms}

Blocking Probability, Optical Network et. al.

\section{Keywords}

Optical network, Blocking probability, Routing Strategies, Erlang's B formula.

\section{INTRODUCTION}

Optical Network provides a key technology in communication networks that provide capabilities for exceeding those of traditional electronic networks. Optical network is used to convey signals over distances by employing any sort of optical technology. In all optical networks, light path (routing path) used unique wavelength during its lifetime [1].

Routing techniques can be classified as Static and Dynamic. In static routing, variation in time does not affect the routing procedure while in dynamic routing, at the time of connection establishment network state information is used [3]. Static routing technique is widely used in fixed routing where each s-d pair assigned a single path. If associated path is not available, call is blocked. In dynamic routing each s-d pair is assigned a set of paths deflective mechanism is used to reroutes a blocked burst from primary to alternative route.

The major applications of the optical networks are video conferencing, scientific visualization, real-time medical imaging, supercomputing, and distributed [2].

Recently different types of optical network were published or under review. In [7] presented by Alexsandra F. Gomes, Carlos E. M. Fernandes, Victor A. P. Oliveira e Iguatemi E. Fonseca presents WDM multiplexing for fiber-optic communications that uses the concept different wavelengths of laser light for multiplexing number of optical carrier signals onto a single optical fiber. Bidirectional communications over a single strand of fiber is possible using this technique. The data is typically carried over $10 \mathrm{Gbps}$ wavelength channels. With the enhancement in hardware used for multiplexing it is possible to multiplex 80-100 channels on a single fiber and more new advances promise to increase these numbers even higher. Besides multiplexers and demultiplexers, other WDM components include fixed and tunable lasers, and continuous-mode and burst-mode receivers.

With rapidly increase in number of users in communication network and services with higher bandwidth requirement leads to data traffic. Therefore, many questions and problems regarding to above parameter are arising like, design \& planning of such networks, performance \& cost evaluation, development of operation \& management concepts etc. OBS technology combines the advantages of both optical circuit switching (OCS) and optical packet switching. OBS is a telecommunication technique that allows dynamic subwavelength switching of data by sending the control signals separately and in advance of the data. The purpose of OBS is to dynamically provision sub-wavelength granularity by optimally combining electronics and optics. As compare to wavelength routing, OBS provides more bandwidth flexibility but requires faster switching and control technology.

No doubt, Optical transmission and switching technologies based on WDM have been increasingly deployed in the Internet infrastructure over the last decade in order to meet the ever increasing demand for bandwidth. Most recently, two trends have emerged in the design and deployment of WDM networks. One is towards increasing transparency in the network so as to eliminate electronic bottlenecks and enable the handling of a broad range of hetero generous signals regardless of protocol formats, bit rates, or modulation [10].

The second trend is towards re-configurability in optical networks, such that bandwidth can be created in real time between end-users to accommodate dynamically changing demands. Optical Packet Switching (OPS) [10] promises almost arbitrarily transmission and switching granularity, evoking the vision of a bandwidth-data-centric all-optical Internet. The realization of this vision, however, faces significant challenges in that OPS requires practical, costeffective, and scalable implementations of optical and packetlevel parsing [11].

We also note that each of the three optical switching technologies (wavelength routing, OBS, and OPS) have important application. Optical Packet Switching domain hence, rather than each technology replacing the previous one, it is highly likely that all three will coexist in the optical network of the future [10]. 


\section{PREVIOUS RESEARCH}

The term optical networks are high capacity telecommunication network. Optical Networks are based on optical technologies and components that provide routing, grooming and restoration at the wavelength level as well as wavelength based services. Hence routing becomes an important issue on these networks means to select best route in the network with minimized blocking probability.

Many routing strategies have been proposed for Optical network by researchers.

In [4] M.Garnot presented three different routing strategies, based on shortest path algorithm, for optical paths to perform allocation of resources (wavelengths) in WDM optical networks. The first routing strategy ("shortest path" routing) is developed for minimizing the connection length and total fiber length. The second routing strategy (LNH) is based on the reduction of the number of hops. The last one routing strategy has been implemented in order to resolve the total disjoined path problem and can be noted heuristic path routing (HPR).

In [5] Xiaowen Chu,Bo Li illustrated weighted leastcongestion routing and first fit wavelength Assignment (WLCR-FF) RWA algorithm. In conjunction with heuristic wavelength converter placement algorithm called MPBF (Minimum Blocking Probability First) that considers both the distribution of free wavelengths and the lengths of each route jointly. The performance of proposed algorithm is studied using both analysis and simulation, and results reflect that in case of proposed WLCR-FF algorithm, blocking performance is better than static routing fixed alternative routing and least loaded routing algorithm in the environment of sparse and full wavelength conversion algorithm [5].

Algorithms based on deflection and converge proposed by D. Barth, which are able to provide high bandwidth and an ending guarantee for the end-to-end delay in a core network, and simulate the proposed work to prove the bounds on the delay [6].

A routing strategy taking into account monitored physical layer attributes as input parameters for the path computation proposed by Alexsandra F. Gomes in [7] known to be Routing and Wavelength Assignment algorithm. The proposed routing technique aims at to achieve optical quality of service $(\mathrm{QoS})$ in optical network impaired by Polarization Mode Dispersion (PMD) which results in improved network performance when alternative routes are used.

In [9] G.Ramesh, Sundara Vadivelu proposed an algorithm, based on concept of load balancing, Reliable and fault tolerant (RFTR) algorithm in order to establish the primary path. Finding a route of light paths for the network with least congestion for source-destination pairs is known to be load balancing. In this proposed algorithm, the traffic is routed over the lightly loaded links [9].

In this paper, a new algorithm is proposed, that is fault tolerant algorithm, for optimization of blocking probability in optical network. The objective of this algorithm is to minimize the blocking probability and to choose the best route.

\section{PROPOSED MATHEMATICAL MODEL}

The proposed mathematical model is designed under the following assumptions:

- Optical network is set of node interconnected by singlefiber link.

- Each fiber link is bi-directional and each link has wavelength channel.

- An array of transmitters and receivers is associated with each Station.

- $\quad$ Number of available wavelength $=10$

- No queuing of connection request. If connection is blocked next alternative path is selected.

- $\quad$ Link loads are independent

For the selection of the alternative path, non-adaptive approach is assumed to be used.

Erlang formula is used to calculate the blocking probability as in equation (2.1). The Erlang is defined as dimensionless unit of traffic intensity. It is dependent on observation time. The maximum that a facility can be in use is $100 \%$ of the time. 1 Erlang is defined as, if the observation time is 10 minutes, and facility is in use for the full time.

$$
\mathrm{R}(N, A, S)=\frac{A^{N}\left(\begin{array}{l}
S \\
N
\end{array}\right)}{\sum^{\mathbb{N}} A^{\mathrm{i}}\left(\begin{array}{l}
S \\
\mathrm{i}
\end{array}\right)}
$$

$A=$ offered traffic intensity in erlangs, from all sources

$\mathrm{S}=$ number of server

$\mathrm{N}=$ number of user

$\mathrm{P}(\mathrm{b})=$ probability of blocking

Algorithm design:-

In proposed algorithm, to transfer data in an optical network $\mathrm{b} / \mathrm{w}$ two nodes, one is selected as source node and other as destination node. All the possible routes are defined $b / w$ source and destination. Here we used dijkstra's algorithm for finding the all-possible paths from the source to the destination by calculating the candidate distance from each node, which is given by

Candidate distance $=$ distance to the solved node + length of $\operatorname{arc}$

A shortest path is select among all paths by first arranging all the path or routes from the source to destination in increasing order. A check is done for fault on the selected path, and if fault exist on this path, select the next path. Among the paths, without faults, blocking probability of every path is calculated using the Erlang formula and the path with minimum blocking probability is selected.

\section{DESCRIPTION OF PROPOSED MATHEMATICAL MODEL}

We consider a WDM network with $\mathrm{N}$ number of nodes, all the nodes forms a set $\mathbf{V}$, and all directed links are contained in a set $\mathbf{E}$. Here we adopt the fixed path routing policy for the light path establishment, the predetermined directed routes are indicated with a set $\mathbf{R}$ and $r \in R$. 
Notations: Upper-case letters are used to denote the path and the network-wide parameters and Upper-case letters are used for link parameters. Subscripts and superscripts refer to specific instances of links, node pairs and routes.

Let us consider the following scenario shown in fig 4.1.

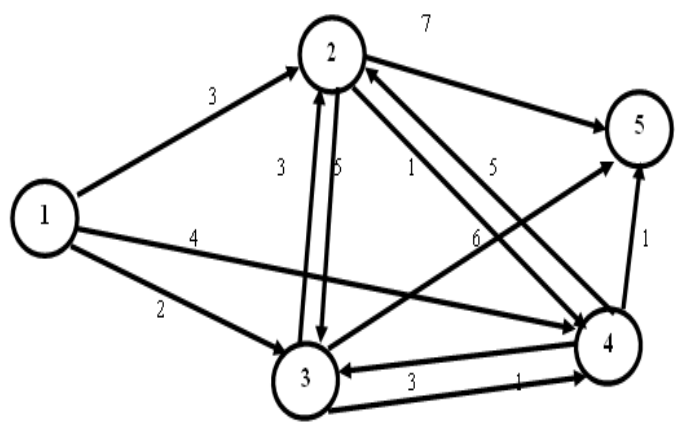

Fig 4.1: Network with the source $s$ and destination $x$ Given

- $\quad \mathbf{N}$ node WDM network

- All the nodes forms a set $\mathbf{V}$.

- $\quad \mathbf{E}$ is the set of all directed links.

- Fixed path routing policy is adopted for the establishment of light path.

- $\quad \mathbf{R}$ indicates the set of predetermined directed routes.

For each route $r \in R$, the blocking probability [4] for the connections along route $r$ can be given by eq. (4.1).

$$
P=1-\left[1-P_{W}\right]^{l}
$$

We can substitute the value of Erlang's loss from Erlang's B formula in eq. (4.1)

$$
\begin{aligned}
& P_{W}=\frac{\frac{L}{W !}}{\sum_{i=0}^{C} \frac{L}{i !}} \\
& P=1-\left[1-\frac{\frac{L}{W !}}{\sum_{i=0}^{C} \frac{L}{i !}}\right]
\end{aligned}
$$

Equation (4.3) is used as mathematical model which can be used for blocking probability calculation in optical networks

\section{SIMULATION AND RESULTS}

For the control of congestion, MATLAB simulator is used. The result from earlier algorithm and new proposed model is compared.

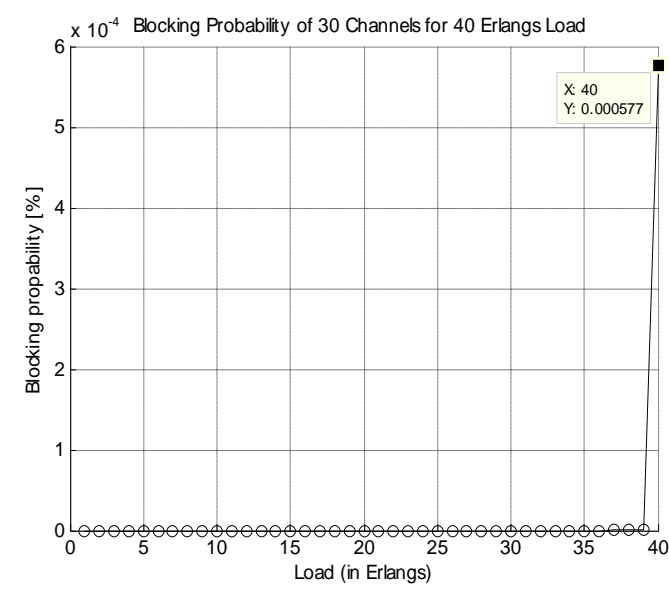

Fig 5.1: Graph showing the Blocking Probability Vs Load for $\mathrm{L}=40, \mathrm{C}=30, \mathrm{P}=10$

In above graph number of channels is less than load on the network. As in this case, numbers of channels are 30 and load on the network is 40 . We have compute the value of blocking probability it varies with different values of load. When load on the network is 5 , blocking probability is 0 .It remains 0 up to load value 35 , as value of load approaches to 40 , it gradually becomes $0.000577 * 10^{-4}$. i.e. approx 0 .

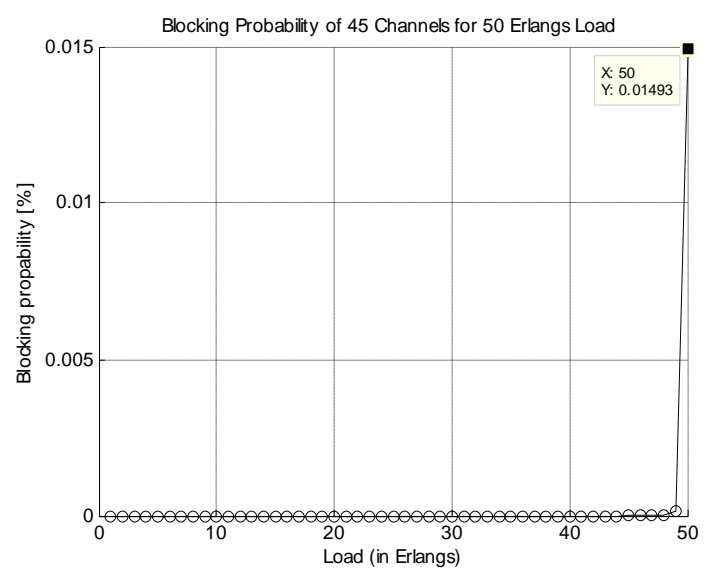

Fig 5.2: Graph showing the Blocking Probability Vs Load for $L=50, C=45, P=5$

Figure 5.2 shows that with the increase in the value of load from 40 to 50 and number of channels from 30 to 45 , the value of blocking probability also increases but negligibly small.



Fig 5.3: Graph showing the Blocking Probability Vs Number of Channels for $L=40, C=30, P=10$ 
In above graph number of channels is less than load on the network. As in this case, number of channels are 30 and load on the network is 40.we have compute the value of blocking probability it varies with different values of no of channels. When no of channels are from 5 to 25 then value of blocking probability is 0 .As value of channel approaches to 30 , it gradually becomes $0.000577 * 10^{-4}$ i.e approx 0 .

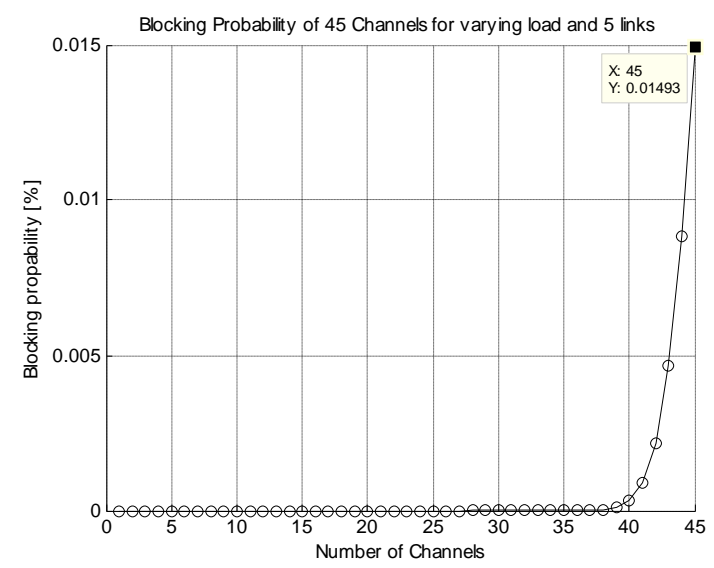

Fig 5.3: Graph showing the Blocking Probability Vs Number of channels for $L=50, C=45, P=5$

Above figure shows that with the increase in the value of load from 40 to 50 and number of channels from 30 to 45 , the value of blocking probability also increases but negligibly small.

\section{CONCLUSION AND FUTURE SCOPE}

In this paper we have minimized the value of blocking probability in optical networks to a minimum value. Earlier the value of the blocking probability was in the range of 10$20 \%$ whereas in this thesis we have reduced this value of blocking probability $0.08025 \%$ negligible to 0 .Hence we can suggest that the proposed model and proposed algorithm are a combination which can be proved better than the conventional algorithms.

In future we can extend our research to work on the different parameters like by enhancing the number of wavelength in the network. We can also extend this work for the infinite number of users. This work can be further applied for the minimization of the blocking probability using different parameters. Random function is not implemented for the detection of the fault only the idea is given in the thesis. The combination of this model and algorithm can be further implemented on different areas and networks such as wireless

\section{REFERENCES}

[1] Rolland Mwanou, Samuel Pierre .cole Polytechnique de Montr.al, QC, "Adaptive Routing Algorithms for AllOptical Networks" IEEE Canadian Review N Fall / Automne 2005

[2] Hong Shen, Member, /€€E, Francis Chin, Fellow, /€E€, and Yi Pan, Senior Member, /€€E, "Efficient FaultTolerant Routing in Multihop Optical WDM Networks", IEEE Transactions On Parallel And Distributed Systems, Vol. No. 10, October 1999.

[3] A.Mokhtar and M.Azizoglu, "Adaptive Wavelength Routing in All-Optical Networks", IEEE/ACM Transactions on Networking, Vol.6, No.2, 197-206, April 1998.

[4] M.Granot,M.Sotom,F.Masetti, "Routing Strategies for Optical Paths in WDM Networks",0-7803-3925-8/97 \$1 0.0001997 IEEE.

[5] XiaowenChu, Bo Li, Kazem Sohraby,Zhensheng Zhang, "Routing and Wavelength Assignment Issues in the Presence of Wavelength Conversion for All-Optical Networks", 0-7803- 7632-3/02/\$17.00 QZOO2 IEEE.

[6] D. Barth, P. Berthomé, D. Chiaroni, J. M. Fourneau, C. Laforest, and S. Vial, "Mixing Convergence and Deflection Strategies for Packet Routing inAll-Optical Networks", VOL. 1, NO. 3/AUGUST 2009/ J. OPT. COMMUN. NETW.

[7] Alexsandra F. Gomes, Carlos E. M. Fernandes, Victor A. P. Oliveira e Iguatemi E. Fonseca "Routing Techniques in Dynamic Optical Networks Impaired by PMD", 978$1-4244-5357-3 / 09 / \$ 26.00 \odot 2009$ IEEE.

[8] Andrzej Szymanski, Artur Lason, Jacek Rzasa, and Andrzej Jajszczyk, "Performance Evaluation of the Grade-of-Service-based Routing Strategies for Optical Networks", 978-1-4244-2075-9/08/\$25.00 @2008 IEEE 5252 .

[9] G.Ramesh, S.SundaraVadivelu 2009, "A Reliable and Fault-Tolerant Routing for Optical WDM Networks", IJCSIS Vol. 6, Issue 2.

[10] (Fiber- cabling handbook).

[11] Tien Van Do,Ram Chakka,Zsolt Pandi, "NOVEL ANALYSIS METHOD FOR OPTICAL PACKET SWITCHING NODES". 